\title{
A Method for Molecular Design Based on Linear Regression and Integer Programming
}

\author{
Jianshen Zhu \\ zhujs@amp.i.kyoto-u.ac.jp \\ Department of Applied Mathematics and \\ Physics, Kyoto University \\ Kyoto, Japan \\ Liang Zhao \\ liang@gsais.kyoto-u.ac.jp \\ Graduate School of Advanced Integrated \\ Studies in Human Survivability \\ (Shishu-Kan), Kyoto University \\ Kyoto, Japan
}

\author{
Naveed A. Azam* \\ azam@amp.i.kyoto-u.ac.jp \\ Department of Applied Mathematics and \\ Physics, Kyoto University \\ Kyoto, Japan \\ Hiroshi Nagamochi \\ nag@amp.i.kyoto-u.ac.jp \\ Department of Applied Mathematics and \\ Physics, Kyoto University \\ Kyoto, Japan
}

\author{
Kazuya Haraguchi \\ haraguchi@amp.i.kyoto-u.ac.jp \\ Department of Applied Mathematics and \\ Physics, Kyoto University \\ Kyoto, Japan \\ Tatsuya Akutsu \\ takutsu@kuicr.kyoto-u.ac.jp \\ Bioinformatics Center, Institute for \\ Chemical Research, Kyoto University \\ Kyoto, Japan
}

\begin{abstract}
Recently a novel framework has been proposed for designing the molecular structure of chemical compounds using both artificial neural networks (ANNs) and mixed integer linear programming (MILP). In the framework, we first define a feature vector $f(\mathbb{C})$ of a chemical graph $\mathbb{C}$ and construct an ANN that maps $x=f(\mathbb{C})$ to a predicted value $\eta(x)$ of a chemical property $\pi$ to $\mathbb{C}$. After this, we formulate an MILP that simulates the computation process of $f(\mathbb{C})$ from $\mathbb{C}$ and that of $\eta(x)$ from $x$. Given a target value $y^{*}$ of the chemical property $\pi$, we infer a chemical graph $\mathbb{C}^{\dagger}$ such that $\eta\left(f\left(\mathbb{C}^{\dagger}\right)\right)=y^{*}$ by solving the MILP. In this paper, we use linear regression to construct a prediction function $\eta$ instead of ANNs. For this, we derive an MILP formulation that simulates the computation process of a prediction function by linear regression. The results of computational experiments suggest our method can infer chemical graphs with around up to 50 non-hydrogen atoms.
\end{abstract}

\section{CCS CONCEPTS}

- Computing methodologies $\rightarrow$ Machine learning; • Mathematics of computing $\rightarrow$ Integer programming; • Applied computing $\rightarrow$ Bioinformatics.

\section{KEYWORDS}

Machine learning, linear regression, integer programming, cheminformatics, materials informatics, QSAR/QSPR, molecular design

\section{ACM Reference Format:}

Jianshen Zhu, Naveed A. Azam, Kazuya Haraguchi, Liang Zhao, Hiroshi Nagamochi, and Tatsuya Akutsu. 2022. A Method for Molecular Design Based on Linear Regression and Integer Programming. In 2022 12th International Conference on Bioscience,

${ }^{*}$ Corresponding author.

This work is licensed under a Creative Commons Attribution International 4.0 License.

ICBBB '22, January 7-10, 2022, Tokyo, Japan

(C) 2022 Copyright held by the owner/author(s).

ACM ISBN 978-1-4503-8738-5/22/01.

https://doi.org/10.1145/3510427.3510431
Biochemistry and Bioinformatics (ICBBB '22), fanuary 7-10, 2022, Tokyo, fapan. ACM, New York, NY, USA, 8 pages. https://doi.org/10.1145/3510427.3510431

\section{INTRODUCTION}

Background Analysis of chemical compounds is an important research topic in biochemistry and bioinformatics because it may lead to the design of novel drugs. Due to its practical importance, various machine learning methods have been applied to the prediction of chemical activities from their structural data, where such a problem is often referred to as quantitative structure activity relationship (QSAR) [11, 19]. Recently, neural networks and deep-learning technologies have extensively been applied to QSAR [5].

In addition to QSAR, extensive studies have been done on inverse quantitative structure activity relationship (inverse QSAR), which seeks for chemical structures having desired chemical activities under some constraints. Since it is difficult to directly handle chemical structures in both QSAR and inverse QSAR, chemical compounds are usually represented as vectors of real or integer numbers, which are often called descriptors in chemoinformatics and correspond to feature vectors in machine learning. One major approach in inverse QSAR is to infer feature vectors from given chemical activities and constraints and then reconstruct chemical structures from these feature vectors $[8,13,14]$, where chemical structures are usually treated as undirected graphs. However, the reconstruction itself is a challenging task because the number of possible chemical graphs is huge. For example, chemical graphs with up to 30 atoms (vertices) C, N, O, and $\mathrm{S}$ may exceed $10^{60}$ [3]. Due to this difficulty, most existing methods for inverse QSAR do not guarantee optimal or exact solutions.

As a new approach, extensive studies have recently been done for inverse QSAR using artificial neural networks (ANNs), especially using graph convolutional networks [9]. For example, recurrent neural networks [15, 21], variational autoencoders [6], grammar variational autoencoders [10], generative adversarial networks [4], and invertible flow models $[12,16]$ have been applied. However, these methods do not yet guarantee optimal or exact solutions.

Framework Akutsu and Nagamochi [1] proved that the computation process of a given ANN can be simulated with a mixed integer linear 


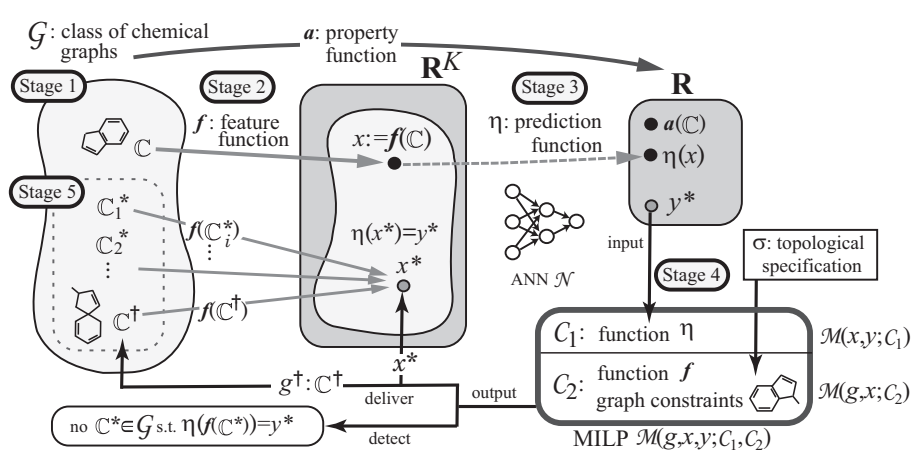

Figure 1: An illustration of a framework for inferring a set of chemical graphs $\mathbb{C}^{*}$.

programming (MILP). Based on this, a novel framework for inferring chemical graphs has been developed [2], as illustrated in Figure 1. It constructs a prediction function in the first phase and infers a chemical graph in the second phase. The first phase of the framework consists of three stages. In Stage 1, we choose a chemical property $\pi$ and a class $\mathcal{G}$ of graphs, where a property function $a$ is defined so that $a(\mathbb{C})$ is the value of $\pi$ for a compound $\mathbb{C} \in \mathcal{G}$, and collect a data set $D_{\pi}$ of chemical graphs in $\mathcal{G}$ such that $a(\mathbb{C})$ is available for every $\mathbb{C} \in D_{\pi}$. In Stage 2, we introduce a feature function $f: \mathcal{G} \rightarrow \mathbb{R}^{K}$ for a positive integer $K$. In Stage 3, we construct a prediction function $\eta$ with an ANN $\mathcal{N}$ that, given a vector $x \in \mathbb{R}^{K}$, returns a value $y=\eta(x) \in \mathbb{R}$ so that $\eta(f(\mathbb{C}))$ serves as a predicted value to the real value $a(\mathbb{C})$ of $\pi$ for each $\mathbb{C} \in D_{\pi}$. Given a target chemical value $y^{*}$, the second phase infers chemical graphs $\mathbb{C}^{*}$ with $\eta\left(f\left(\mathbb{C}^{*}\right)\right)=y^{*}$ in the next two stages. We have obtained a feature function $f$ and a prediction function $\eta$ and call an additional constraint on the substructures of target chemical graphs a topological specification. In Stage 4, we prepare the following two MILP formulations:

- MILP $\mathcal{M}\left(x, y ; C_{1}\right)$ with a set $C_{1}$ of linear constraints on variables $x$ and $y$ (and some other auxiliary variables) simulates the process of computing $y:=\eta(x)$ from a vector $x$; and

- MILP $\mathcal{M}\left(g, x ; C_{2}\right)$ with a set $C_{2}$ of linear constraints on variable $x$ and a variable vector $g$ that represents a chemical graph $\mathbb{C}$ (and some other auxiliary variables) simulates the process of computing $x:=f(\mathbb{C})$ from a chemical graph $\mathbb{C}$ and chooses a chemical graph $\mathbb{C}$ that satisfies the given topological specification $\sigma$.

Given a target value $y^{*} \in \mathbb{R}$, we solve the combined MILP $\mathcal{M}\left(g, x, y ; C_{1}, C_{2}\right)$ to find a feature vector $x^{*} \in \mathbb{R}^{K}$ and a chemical graph $\mathbb{C}^{\dagger}$ with the specification $\sigma$ such that $f\left(\mathbb{C}^{\dagger}\right)=x^{*}$ and $\eta\left(x^{*}\right)=y^{*}$ (where if the MILP instance is infeasible then this suggests that there does not exist such a desired chemical graph). In Stage 5, we generate other chemical graphs $\mathbb{C}^{*}$ such that $\eta\left(f\left(\mathbb{C}^{*}\right)\right)=y^{*}$ based on the output chemical graph $\mathbb{C}^{\dagger}$.

Two-layered Model Recently Shi et al. [17] proposed a new model, called a two-layered model for representing the feature of a chemical graph in order to deal with an arbitrary graph in the framework and refined the set of rules for describing a topological specification so that a prescribed structure can be included in both of the acyclic and cyclic parts of $\mathbb{C}$. In the two-layered model, a chemical graph $\mathbb{C}$ with a parameter $\rho \geq 1$ is regarded as two parts: the exterior and the interior of the hydrogensuppressed chemical graph $\langle\mathbb{C}\rangle$ obtained from $\mathbb{C}$ by removing hydrogen. The exterior consists of maximal acyclic induced subgraphs with height at most $\rho$ in $\langle\mathbb{C}\rangle$ and the interior is the connected subgraph of $\langle\mathbb{C}\rangle$ obtained by ignoring the exterior. Shi et al. [17] defined a feature vector $f(\mathbb{C})$ of a chemical graph $\mathbb{C}$ to be a combination of the frequency of adjacent atom pairs in the interior and the frequency of chemical acyclic graphs among the set of chemical rooted trees $T_{u}$ rooted at interior-vertices $u$. Recently, Tanaka et al. [18] extended the model to treat a chemical graph with hydrogens directly so that more variety of chemical rooted trees represent the feature of the exterior.

Contribution In this paper, we first make a slight but chemically important modification to a model of chemical graphs proposed by Tanaka et al. [18] so that we can treat a chemical element with multi-valence such as sulfur $\mathrm{S}$ and a chemical graph with cations and anions.

The quality of a prediction function $\eta$ constructed in Stage 3 is one of the most important factors in the framework. It is also pointed out that overfitting is a major issue in ANN-based approaches for QSAR because ANNs have many parameters to be optimized [5]. Tanaka et al. [18] observed that decision trees perform better than ANNs for some chemical properties and used a decision tree for constructing a prediction function $\eta$ in Stage 3. In this paper, we use linear regression to construct a prediction function in Stage 3. Linear regression is much simpler than ANNs and decision trees and thereby we regard the performance of a prediction function by linear regression as the basis for other more sophisticated machine learning methods. In this paper, we derive an MILP formulation $\mathcal{M}\left(x, y ; C_{1}\right)$ that simulates the computation process of a prediction function by linear regression. For an MILP formulation $\mathcal{M}\left(g, x ; C_{2}\right)$ that represents a feature function $f$ and a specification $\sigma$ in Stage 4, we can use the same formulation proposed by Tanaka et al. [18] with a slight modification (the detail of the MILP $\mathcal{M}\left(g, x ; C_{2}\right)$ can be found in a full preprint version [22]). To generate target chemical graphs $\mathbb{C}^{*}$ in Stage 5, we can also use the dynamic programming algorithm due to Tanaka et al. [18] with a slight modification and omit the details in this paper.

We implemented the framework based on the refined two-layered model and a prediction function by linear regression. The results of our computational experiments reveal a set of chemical properties to which a prediction function constructed with linear regression on our feature function performs well. We also observe that the proposed method can infer chemical graphs with up to 50 non-hydrogen atoms.

The paper is organized as follows. Section 2 introduces some notions on graphs, a modeling of chemical compounds and a choice of descriptors. Section 3 describes our modification to the two-layered model. Section 4 reviews the idea of linear regression and formulates an $\operatorname{MILP} \mathcal{M}\left(x, y ; C_{1}\right)$ that simulates a process of computing a prediction function constructed by linear regression. Section 5 reports the results of some computational experiments that were conducted for 18 chemical properties such as vapor density and optical rotation. Section 6 makes some concluding remarks. Some technical details are given in a full preprint version [22].

\section{PRELIMINARY}

This section introduces some notions and terminologies on graphs, modeling of chemical compounds and our choice of descriptors. 
Let $\mathbb{R}, \mathbb{R}_{+}, \mathbb{Z}$ and $\mathbb{Z}_{+}$denote the sets of reals, non-negative reals, integers and non-negative integers, respectively. For two integers $a$ and $b$, let $[a, b]$ denote the set of integers $i$ with $a \leq i \leq b$.

Graph Given a graph $G$, let $V(G)$ and $E(G)$ denote the sets of vertices and edges, respectively. For a subset $V^{\prime} \subseteq V(G)$ (resp., $E^{\prime} \subseteq E(G)$ ) of a graph $G$, let $G-V^{\prime}$ (resp., $G-E^{\prime}$ ) denote the graph obtained from $G$ by removing the vertices in $V^{\prime}$ (resp., the edges in $E^{\prime}$ ), where we remove all edges incident to a vertex in $V^{\prime}$ in $G-V^{\prime}$. An edge subset $E^{\prime} \subseteq E(G)$ in a connected graph $G$ is called separating (resp., non-separating) if $G-E^{\prime}$ becomes disconnected (resp., $G-E^{\prime}$ remains connected). The $\operatorname{rank} \mathrm{r}(G)$ of a graph $G$ is defined to be the minimum $|F|$ of an edge subset $F \subseteq E(G)$ such that $G-F$ contains no cycle, where $\mathrm{r}(G)=|E(G)|-|V(G)|+1$. Observe that $\mathrm{r}\left(G-E^{\prime}\right)=\mathrm{r}(G)-\left|E^{\prime}\right|$ holds for any non-separating edge subset $E^{\prime} \subseteq E(G)$. An edge $e=u_{1} u_{2} \in E(G)$ in a connected graph $G$ is called a bridge if $\{e\}$ is separating, i.e., $G-e$ consists of two connected graphs $G_{i}$ containing vertex $u_{i}, i=1,2$. For a connected cyclic graph $G$, an edge $e$ is called a core-edge if it is in a cycle of $G$ or is a bridge $e=u_{1} u_{2}$ such that each of the connected graphs $G_{i}, i=1,2$ of $G-e$ contains a cycle. A vertex incident to a core-edge is called a core-vertex of $G$. A path with two end-vertices $u$ and $v$ is called a $u, v$-path. A vertex designated in a graph $G$ is called a root.

For a graph $G$ possibly with a root, a leaf-vertex is defined to be a nonroot vertex with degree 1 , call the edge $u v$ incident to a leaf vertex $v$ a leaf-edge, and denote by $V_{\text {leaf }}(G)$ and $E_{\text {leaf }}(G)$ the sets of leaf-vertices and leaf-edges in $G$, respectively. For a graph or a rooted graph $G$, we define graphs $G_{i}, i \in \mathbb{Z}_{+}$obtained from $G$ by removing the set of leaf-vertices $i$ times so that $G_{0}:=G ; G_{i+1}:=G_{i}-V_{\text {leaf }}\left(G_{i}\right)$, where we call a vertex $v \in V_{\text {leaf }}\left(G_{k}\right)$ a leaf $k$-branch and we say that a vertex $v \in V_{\text {leaf }}\left(G_{k}\right)$ has height $h \mathrm{t}(v)=k$ in $G$. The height $\mathrm{ht}(T)$ of a rooted tree $T$ is defined to be the maximum of $h t(v)$ of a vertex $v \in V(T)$. For an integer $k \geq 0$, we call a rooted tree $T k$-lean if $T$ has at most one leaf $k$-branch.

\subsection{Modeling of Chemical Compounds}

To represent a chemical compound, we introduce a set of chemical elements such as $\mathrm{H}$ (hydrogen), C (carbon), $\mathrm{O}$ (oxygen), $\mathrm{N}$ (nitrogen) and so on. To distinguish a chemical element a with multiple valences such as $\mathrm{S}$ (sulfur), we denote a chemical element a with a valence $i$ by $\mathrm{a}_{(i)}$, where we do not use such a suffix $(i)$ for a chemical element a with a unique valence. Let $\Lambda$ be a set of chemical elements $\mathrm{a}_{(i)}$. For example, $\Lambda=\left\{\mathrm{H}, \mathrm{C}, \mathrm{O}, \mathrm{N}, \mathrm{P}, \mathrm{S}_{(2)}, \mathrm{S}_{(4)}, \mathrm{S}_{(6)}\right\}$. Let val : $\Lambda \rightarrow[1,6]$ be a valence function. For example, $\operatorname{val}(\mathrm{H})=1, \operatorname{val}(\mathrm{C})=4, \operatorname{val}(0)=2, \operatorname{val}(\mathrm{P})=5, \operatorname{val}\left(\mathrm{S}_{(2)}\right)=2$, $\operatorname{val}\left(\mathrm{S}_{(4)}\right)=4$ and $\operatorname{val}\left(\mathrm{S}_{(6)}\right)=6$. For each chemical element $\mathrm{a} \in \Lambda$, let mass(a) denote the mass of a.

A chemical compound is represented by a chemical graph defined to be a tuple $\mathbb{C}=(H, \alpha, \beta)$ of a simple, connected undirected graph $H$ and functions $\alpha: V(H) \rightarrow \Lambda$ and $\beta: E(H) \rightarrow[1,3]$. The set of atoms and the set of bonds in the compound are represented by the vertex set $V(H)$ and the edge set $E(H)$, respectively. The chemical element assigned to a vertex $v \in V(H)$ is represented by $\alpha(v)$ and the bond-multiplicity between two adjacent vertices $u, v \in V(H)$ is represented by $\beta(e)$ of the edge $e=u v \in E(H)$.

For a notational convenience, we use a function $\beta_{\mathbb{C}}: V(H) \rightarrow[0,12]$ for a chemical graph $\mathbb{C}=(H, \alpha, \beta)$ such that $\beta_{\mathbb{C}}(u)$ means the sum of bondmultiplicities of edges incident to a vertex $u$; i.e., $\beta_{\mathbb{C}}(u) \triangleq \sum_{u v \in E(H)} \beta(u v)$ for each vertex $u \in V(H)$. For each vertex $u \in V(H)$, define the electrondegree eledeg $_{\mathbb{C}}(u)$ to be eledeg $\operatorname{Cos}_{\mathbb{C}}(u) \triangleq \beta_{\mathbb{C}}(u)-\operatorname{val}(\alpha(u))$. For each vertex $u \in V(H)$, let $\operatorname{deg}_{\mathbb{C}}(u)$ denote the number of vertices adjacent to the vertex $u$ in $\mathbb{C}$.

For a chemical graph $\mathbb{C}=(H, \alpha, \beta)$, let $V_{a}(\mathbb{C})$, a $\in \Lambda$ denote the set of vertices $v \in V(H)$ such that $\alpha(v)=a$ in $\mathbb{C}$ and define the hydrogensuppressed chemical graph $\langle\mathbb{C}\rangle$ to be the graph obtained from $H$ by removing all the vertices $v \in V_{\mathrm{H}}(\mathbb{C})$.

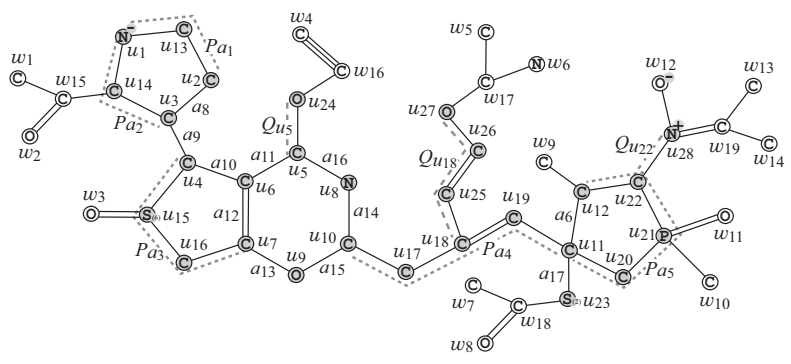

Figure 2: An illustration of a hydrogen-suppressed chemical graph $\langle\mathbb{C}\rangle$ obtained from a chemical graph $\mathbb{C}$ with $\mathrm{r}(\mathbb{C})=4$ by removing all the hydrogens, where for $\rho=2, V^{\operatorname{ex}}(\mathbb{C})=\left\{w_{i} \mid i \in\right.$ $[1,19]\}$ and $V^{\text {int }}(\mathbb{C})=\left\{u_{i} \mid i \in[1,28]\right\}$.

\section{TWO-LAYERED MODEL}

This section reviews the two-layered model and describes our modification to the model.

Let $\mathbb{C}=(H, \alpha, \beta)$ be a chemical graph and $\rho \geq 1$ be an integer, which we call a branch-parameter.

A two-layered model of $\mathbb{C}$ is a partition of the hydrogen-suppressed chemical graph $\langle\mathbb{C}\rangle$ into an "interior" and an "exterior" in the following way. We call a vertex $v \in V(\langle\mathbb{C}\rangle)$ (resp., an edge $e \in E(\langle\mathbb{C}\rangle)$ ) of $G$ an exterior-vertex (resp., exterior-edge) if $h t(v)<\rho$ (resp., $e$ is incident to an exterior-vertex) and denote the sets of exterior-vertices and exterior-edges by $V^{\mathrm{ex}}(\mathbb{C})$ and $E^{\mathrm{ex}}(\mathbb{C})$, respectively, and denote $V^{\text {int }}(\mathbb{C})=V(\langle\mathbb{C}\rangle) \backslash V^{\mathrm{ex}}(\mathbb{C})$ and $E^{\text {int }}(\mathbb{C})=E(\langle\mathbb{C}\rangle) \backslash E^{\text {ex }}(\mathbb{C})$, respectively. We call a vertex in $V^{\text {int }}(\mathbb{C})$ (resp., an edge in $E^{\text {int }}(\mathbb{C})$ ) an interior-vertex (resp., interior-edge). The set $E^{\mathrm{ex}}(\mathbb{C})$ of exterior-edges forms a collection of connected graphs each of which is regarded as a rooted tree $T$ rooted at the vertex $v \in V(T)$ with the maximum $h t(v)$. Let $\mathcal{T}^{\mathrm{ex}}(\langle\mathbb{C}\rangle)$ denote the set of these chemical rooted trees in $\langle\mathbb{C}\rangle$. The interior $\mathbb{C}^{\text {int }}$ of $\mathbb{C}$ is defined to be the subgraph $\left(V^{\text {int }}(\mathbb{C}), E^{\text {int }}(\mathbb{C})\right)$ of $\langle\mathbb{C}\rangle$.

Figure 2 illustrates an example of a hydrogen-suppressed chemical graph $\langle\mathbb{C}\rangle$. For a branch-parameter $\rho=2$, the interior of the chemical graph $\langle\mathbb{C}\rangle$ in Figure 2 is obtained by removing the set of vertices with degree $1 \rho=2$ times; i.e., first remove the set $V_{1}=\left\{w_{1}, w_{2}, \ldots, w_{14}\right\}$ of vertices of degree 1 in $\langle\mathbb{C}\rangle$ and then remove the set $V_{2}=\left\{w_{15}, w_{16}, \ldots, w_{19}\right\}$ of vertices of degree 1 in $\langle\mathbb{C}\rangle-V_{1}$, where the removed vertices become the exterior-vertices of $\langle\mathbb{C}\rangle$.

For each interior-vertex $u \in V^{\text {int }}(\mathbb{C})$, let $T_{u} \in \mathcal{T}^{\mathrm{ex}}(\langle\mathbb{C}\rangle)$ denote the chemical tree rooted at $u$ (where possibly $T_{u}$ consists of vertex $u$ ) and define the $\rho$-fringe-tree $\mathbb{C}[u]$ to be the chemical rooted tree obtained from $T_{u}$ by putting back the hydrogens originally attached with $T_{u}$ in $\mathbb{C}$. Let 
$\mathcal{T}(\mathbb{C})$ denote the set of $\rho$-fringe-trees $\mathbb{C}[u], u \in V^{\text {int }}(\mathbb{C})$. Figure 3 illustrates the set $\mathcal{T}(\mathbb{C})=\left\{\mathbb{C}\left[u_{i}\right] \mid i \in[1,28]\right\}$ of the 2 -fringe-trees of the example $\mathbb{C}$ in Figure 2.

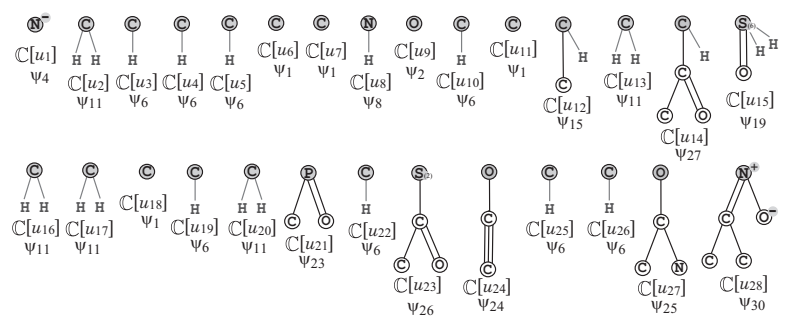

Figure 3: The set $\mathcal{T}(\mathbb{C})$ of 2-fringe-trees $\mathbb{C}\left[u_{i}\right], i \in[1,28]$ of $\mathbb{C}$ with $\langle\mathbb{C}\rangle$ in Figure 2, where the root of each tree is depicted with a gray circle and the hydrogens attached to non-root vertices are omitted in the figure.

Feature Function The feature of an interior-edge $e=u v \in E^{\text {int }}(\mathbb{C})$ such that $\alpha(u)=\mathrm{a}, \operatorname{deg}_{\langle\mathbb{C}\rangle}(u)=d, \alpha(v)=\mathrm{b}, \operatorname{deg}_{\langle\mathbb{C}\rangle}(v)=d^{\prime}$ and $\beta(e)=m$ is represented by a tuple $\left(\mathrm{a} d, \mathrm{~b} d^{\prime}, m\right)$, which is called the edge-configuration of the edge $e$, where we call the tuple $(\mathrm{a}, \mathrm{b}, m)$ the adjacency-configuration of the edge $e$.

For an integer $K$, a feature vector $f(\mathbb{C})$ of a chemical graph $\mathbb{C}$ is defined by a feature function $f$ that consists of $K$ descriptors. We call $\mathbb{R}^{K}$ the feature space.

Tanaka et al. [18] defined a feature vector $f(\mathbb{C}) \in \mathbb{R}^{K}$ to be a combination of the frequency of edge-configurations of the interior-edges and the frequency of chemical rooted trees among the set of chemical rooted trees $\mathbb{C}[u]$ for all interior-vertices $u$. In this paper, we introduce the rank and the adjacency-configuration of leaf-edges as new descriptors in a feature vector of a chemical graph.

Topological Specification A topological specification is described as a set of the following rules proposed by Shi et al. [17] and modified by Tanaka et al. [18]:

(i) a seed graph $G_{\mathrm{C}}$ as an abstract form of a target chemical graph $\mathbb{C}$;

(ii) a set $\mathcal{F}$ of chemical rooted trees as candidates for a tree $\mathbb{C}[u]$ rooted at each exterior-vertex $u$ in $\mathbb{C}$; and

(iii) lower and upper bounds on the number of components in a target chemical graph such as chemical elements, double/triple bonds and the interior-vertices in $\mathbb{C}$.

Figure 4(a) and (b) illustrate examples of a seed graph $G_{C}$ and a set $\mathcal{F}$ of chemical rooted trees, respectively. Given a seed graph $G_{C}$, the interior of a target chemical graph $\mathbb{C}$ is constructed from $G_{C}$ by replacing some edges $a=u v$ with paths $P_{a}$ between the end-vertices $u$ and $v$ and by attaching new paths $Q_{v}$ to some vertices $v$. For example, a chemical graph $\langle\mathbb{C}\rangle$ in Figure 2 is constructed from the seed graph $G_{C}$ in Figure $4(a)$ as follows.

- First replace five edges $a_{1}=u_{1} u_{2}, a_{2}=u_{1} u_{3}, a_{3}=u_{4} u_{7}, a_{4}=u_{10} u_{11}$ and $a_{5}=u_{11} u_{12}$ in $G_{C}$ with new paths $P_{a_{1}}=\left(u_{1}, u_{13}, u_{2}\right), P_{a_{2}}=$ $\left(u_{1}, u_{14}, u_{3}\right), P_{a_{3}}=\left(u_{4}, u_{15}, u_{16}, u_{7}\right), P_{a_{4}}=\left(u_{10}, u_{17}, u_{18}, u_{19}, u_{11}\right)$ and $P_{a_{5}}=\left(u_{11}, u_{20}, u_{21}, u_{22}, u_{12}\right)$, respectively to obtain a subgraph $G_{1}$ of $\langle\mathbb{C}\rangle$.
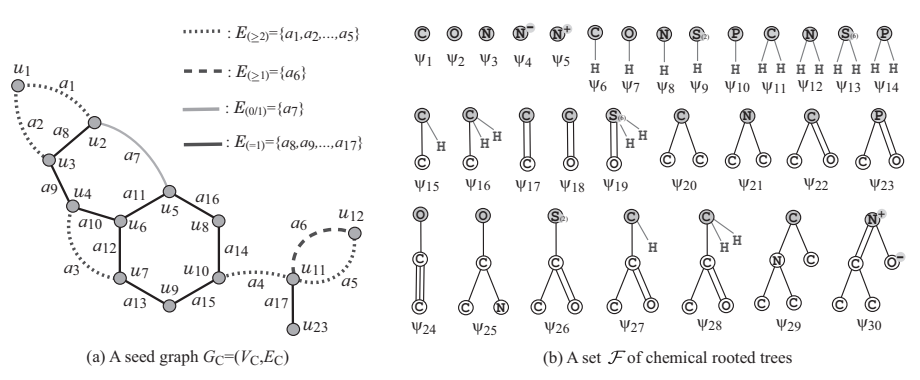

Figure 4: (a) An illustration of a seed graph $G_{C}$ with $r\left(G_{C}\right)=5$ where the vertices in $V_{C}$ are depicted with gray circles, the edges in $E_{(\geq 2)}$ are depicted with dotted lines, the edges in $E_{(\geq 1)}$ are depicted with dashed lines, the edges in $E_{(0 / 1)}$ are depicted with gray bold lines and the edges in $E_{(=1)}$ are depicted with black solid lines; (b) A set $\mathcal{F}=\left\{\psi_{1}, \psi_{2}, \ldots, \psi_{30}\right\} \subseteq \mathcal{F}\left(D_{\pi}\right)$ of 30 chemical rooted trees $\psi_{i}, i \in[1,30]$, where the root of each tree is depicted with a gray circle, where the hydrogens attached to non-root vertices are omitted in the figure.

- Next attach to this graph $G_{1}$ three new paths $Q_{u_{5}}=\left(u_{5}, u_{24}\right), Q_{u_{18}}=$ $\left(u_{18}, u_{25}, u_{26}, u_{27}\right)$ and $Q_{u_{22}}=\left(u_{22}, u_{28}\right)$ to obtain the interior of $\langle\mathbb{C}\rangle$ in Figure 2.

- Finally attach to the interior 28 trees selected from the set $\mathcal{F}$ and assign chemical elements and bond-multiplicities in the interior to obtain a chemical graph $\mathbb{C}$ in Figure 2. In Figure 3, $\psi_{1} \in \mathcal{F}$ is selected for $\mathbb{C}\left[u_{i}\right], i \in\{6,7,11\}$. Similarly $\psi_{2}$ for $\mathbb{C}\left[u_{9}\right], \psi_{4}$ for $\mathbb{C}\left[u_{1}\right], \psi_{6}$ for $\mathbb{C}\left[u_{i}\right], i \in\{3,4,5,10,19,22,25,26\}, \psi_{8}$ for $\mathbb{C}\left[u_{8}\right], \psi_{11}$ for $\mathbb{C}\left[u_{i}\right], i \in$ $\{2,13,16,17,20\}, \psi_{15}$ for $\mathbb{C}\left[u_{12}\right], \psi_{19}$ for $\mathbb{C}\left[u_{15}\right], \psi_{23}$ for $\mathbb{C}\left[u_{21}\right], \psi_{24}$ for $\mathbb{C}\left[u_{24}\right], \psi_{25}$ for $\mathbb{C}\left[u_{27}\right], \psi_{26}$ for $\mathbb{C}\left[u_{23}\right], \psi_{27}$ for $\mathbb{C}\left[u_{14}\right]$ and $\psi_{30}$ for $\mathbb{C}\left[u_{28}\right]$.

Our definition of a topological specification is analogous with the one by Tanaka et al. [18] except for a necessary modification due to the introduction of multiple valences of chemical elements, cations and anions (see a full preprint version [22] for a full description of topological specification).

\section{LINEAR REGRESSIONS}

For an integer $p \geq 1$ and a vector $x \in \mathbb{R}^{p}$, the $j$-th entry of $x$ is denoted by $x(j), j \in[1, p]$.

Let $D$ be a data set of chemical graphs $\mathbb{C}$ with an observed value $a(\mathbb{C}) \in \mathbb{R}$, where we denote by $a_{i}=a\left(\mathbb{C}_{i}\right)$ for an indexed graph $\mathbb{C}_{i}$.

Let $f$ be a feature function that maps a chemical graph $\mathbb{C}$ to a vector $f(\mathbb{C}) \in \mathbb{R}^{K}$ where we denote by $x_{i}=f\left(\mathbb{C}_{i}\right)$ for an indexed graph $\mathbb{C}_{i}$. For a prediction function $\eta: \mathbb{R}^{K} \rightarrow \mathbb{R}$, define an error function

$$
\operatorname{Err}(\eta ; D) \triangleq \sum_{\mathbb{C}_{i} \in D}\left(a_{i}-\eta\left(f\left(\mathbb{C}_{i}\right)\right)\right)^{2}=\sum_{\mathbb{C}_{i} \in D}\left(a_{i}-\eta\left(x_{i}\right)\right)^{2},
$$

and define the coefficient of determination $\mathrm{R}^{2}(\eta, D)$ to be

$$
\mathrm{R}^{2}(\eta, D) \triangleq 1-\frac{\operatorname{Err}(\eta ; D)}{\sum_{\mathbb{C}_{i} \in D}\left(a_{i}-\widetilde{a}\right)^{2}} \text { for } \tilde{a}=\frac{1}{|D|} \sum_{\mathbb{C} \in D} a(\mathbb{C}) .
$$


For a feature space $\mathbb{R}^{K}$, a hyperplane is defined to be a pair $(w, b)$ of a vector $w \in \mathbb{R}^{K}$ and a real $b \in \mathbb{R}$. Given a hyperplane $(w, b) \in \mathbb{R}^{K+1}$, a prediction function $\eta_{w, b}: \mathbb{R}^{K} \rightarrow \mathbb{R}$ is defined by setting $\eta_{w, b}(x) \triangleq$ $w \cdot x+b=\sum_{j \in[1, K]} w(j) x(j)+b$. We can observe that such a prediction function can be represented as an ANN with an input layer with $K$ nodes $u_{j}, j \in[1, K]$ and an output layer with a single node $v$ such that the weight of edge $\operatorname{arc}\left(u_{j}, v\right)$ is set to be $w(j)$, the bias of node $u$ is set to be $b$ and the activation function at node $u$ is set to be a linear function. However, a learning algorithm for an ANN may not find a set of weights $w(j), j \in[1, K]$ and $b$ that minimizes the error function, since the algorithm simply iterates modification of the current weights and biases until it terminates at a local optima in the minimization.

We wish to find a hyperplane $(w, b)$ that minimizes the error function $\operatorname{Err}\left(\eta_{w, b} ; D\right)$. In many cases, a feature vector $f$ contains descriptors that do not play an essential role in constructing a good prediction function. When we solve the minimization problem, the entries $w(j)$ for some descriptors $j \in[1, K]$ in the resulting hyperplane $(w, b)$ become zero, which means that these descriptors were not necessarily important for finding a prediction function $\eta_{w, b}$. It is proposed that solving the minimization with an additional penalty term $\tau$ to the error function often results in a more number of entries $w(j)=0$, reducing a set of descriptors necessary for defining a prediction function $\eta_{w, b}$. For an error function with such a penalty term, a Ridge function $\frac{1}{2|D|} \operatorname{Err}\left(\eta_{w, b} ; D\right)+\lambda\left[\sum_{j \in[1, K]} w(j)^{2}+b^{2}\right]$ [7] and a Lasso function $\frac{1}{2|D|} \operatorname{Err}\left(\eta_{w, b} ; D\right)+\lambda\left[\sum_{j \in[1, K]}|w(j)|+|b|\right][20]$ are known, where $\lambda \in \mathbb{R}$ is a given real number.

Given a prediction function $\eta_{w, b}$, we can simulate a process of computing the output $\eta_{w, b}(x)$ for an input $x \in \mathbb{R}^{K}$ as an MILP $\mathcal{M}\left(x, y ; C_{1}\right)$ in the framework. By solving such an MILP for a specified target value $y^{*}$, we can find a vector $x^{*} \in \mathbb{R}^{K}$ such that $\eta_{w, b}\left(x^{*}\right)=y^{*}$. Instead of specifying a single target value $y^{*}$, we use lower and upper bounds $y^{*}, \bar{y}^{*} \in \mathbb{R}$ on the value $a(\mathbb{C})$ of a chemical graph $\mathbb{C}$ to be inferred. We can control the range between $y^{*}$ and $\bar{y}^{*}$ for searching a chemical graph $\mathbb{C}$ by setting $y^{*}$ and $\bar{y}^{*}$ to be close or different values. A desired MILP is formulated as follows.

$\mathcal{M}\left(x, y ; C_{1}\right):$ An MILP formulation for the inverse problem to prediction function.

\section{constants:}

- A hyperplane $(w, b)$ with $w \in \mathbb{R}^{K}$ and $b \in \mathbb{R}$;

- Real values $y^{*}, \bar{y}^{*} \in \mathbb{R}$ such that $y^{*}<\bar{y}^{*}$;

- A set $I_{\mathbb{Z}}$ of indices $j \in[1, K]$ such that the $j$-th descriptor $\operatorname{dcp}_{j}(\mathbb{C})$ is always an integer;

- A set $I_{+}$of indices $j \in[1, K]$ such that the $j$-th descriptor $\operatorname{dcp}_{j}(\mathbb{C})$ is always non-negative;

- $\ell(j), u(j) \in \mathbb{R}, j \in[1, K]:$ lower and upper bounds on the $j$-th descriptor;

\section{variables:}

- Non-negative integer variable $x(j) \in \mathbb{Z}_{+}, j \in I_{\mathbb{Z}} \cap I_{+}$;

- Integer variable $x(j) \in \mathbb{Z}, j \in I_{\mathbb{Z}} \backslash I_{+}$;

- Non-negative real variable $x(j) \in \mathbb{Z}_{+}, j \in I_{+} \backslash I_{\mathbb{Z}}$;

- Real variable $x(j) \in \mathbb{Z}, j \in[1, K] \backslash\left(I_{\mathbb{Z}} \cup I_{+}\right)$;

\section{constraints:}

$$
\ell(j) \leq x(j) \leq u(j), j \in[1, K], \underline{y}^{*} \leq \sum_{j \in[1, K]} w(j) x(j)+b \leq \bar{y}^{*} .
$$

The number of variables and constraints in the above MILP formulation is $O(K)$. It is not difficult to see that the above MILP is an NP-hard problem.

The entire MILP for Stage 4 consists of the two MILPs $\mathcal{M}\left(x, y ; C_{1}\right)$ and $\mathcal{M}\left(g, x ; C_{2}\right)$ with no objective function. The latter represents the computation process of our feature function $f$ and a given topological specification. See a full preprint version [22] for the details of MILP $\mathcal{M}\left(g, x ; C_{2}\right)$.

\section{RESULTS}

We implemented our method of Stages 1 to 5 for inferring chemical graphs under a given topological specification and conducted experiments to evaluate the computational efficiency. We executed the experiments on a PC with Processor: Core i7-9700 (3.0 GHz; $4.7 \mathrm{GHz}$ at the maximum) and Memory: 16 GB RAM DDR4.

Results on Phase 1. We have conducted experiments of linear regression for 37 chemical properties among which we report the following 18 properties to which the test coefficient of determination $\mathrm{R}^{2}$ attains at least 0.8: octanol/water partition coefficient (Kow), heat of combustion (Hc), vapor density (VD), optical rotation (OpTR), electron density on the most positive atom (EDPA), melting point (MP), heat of atomization $(\mathrm{HA})$, heat of formation $(\mathrm{HF})$, internal energy at $0 \mathrm{~K}(\mathrm{U} 0)$, energy of lowest unoccupied molecular orbital (LUMO), isotropic polarizability (ALPHA), heat capacity at $298.15 \mathrm{~K}(\mathrm{CV})$, solubility (SL), surface tension (SFT), viscosity (VIS), isobaric heat capacities in liquid phase (IHCLIQ), isobaric heat capacities in solid phase (IHCSol) and lipophilicity (LP).

We implemented Stages 1, 2 and 3 in Phase 1 as follows.

Stage 1. We set a graph class $\mathcal{G}$ to be the set of all chemical graphs with any graph structure, and set a branch-parameter $\rho$ to be 2 .

For each of the properties, we first select a set $\Lambda$ of chemical elements and then collect a data set $D_{\pi}$ on chemical graphs over the set $\Lambda$ of chemical elements. To construct the data set $D_{\pi}$, we eliminated chemical compounds that do not satisfy one of the following: the graph is connected, the number of carbon atoms is at least four, and the number of nonhydrogen neighbors of each atom is at most 4 .

Table 1 shows the size and range of data sets that we prepared for each chemical property in Stage 1, where we denote the following:

- $\Lambda$ : the set of elements used in the data set $D_{\pi} ; \Lambda$ is one of the following 11 sets:

$\Lambda_{1}=\{\mathrm{H}, \mathrm{C}, \mathrm{O}\} ; \Lambda_{2}=\{\mathrm{H}, \mathrm{C}, \mathrm{O}, \mathrm{N}\} ; \Lambda_{3}=\{\mathrm{H}, \mathrm{C}, \mathrm{O}, \mathrm{S}(2)\} ; \Lambda_{4}=\{\mathrm{H}, \mathrm{C}, \mathrm{O}, \mathrm{Si}\} ;$ $\Lambda_{5}=\left\{\mathrm{H}, \mathrm{C}, \mathrm{O}, \mathrm{N}, \mathrm{Cl}, \mathrm{P}_{(3)}, \mathrm{P}_{(5)}\right\} ; \Lambda_{6}=\left\{\mathrm{H}, \mathrm{C}, \mathrm{O}, \mathrm{N}, \mathrm{S}_{(2)}, \mathrm{F}\right\} ; \Lambda_{7}=\{\mathrm{H}, \mathrm{C}, \mathrm{O}, \mathrm{N}$, $\left.\mathrm{S}_{(2)}, \mathrm{S}_{(6)}, \mathrm{Cl}\right\} ; \Lambda_{8}=\left\{\mathrm{H}, \mathrm{C}_{(2)}, \mathrm{C}_{(3)}, \mathrm{C}_{(4)}, \mathrm{O}, \mathrm{N}_{(2)}, \mathrm{N}_{(3)}\right\} ; \Lambda_{9}=\left\{\mathrm{H}, \mathrm{C}, \mathrm{O}, \mathrm{N}, \mathrm{S}_{(2)}\right.$, $\left.\mathrm{S}_{(4)}, \mathrm{S}_{(6)}, \mathrm{Cl}\right\} ; \Lambda_{10}=\left\{\mathrm{H}, \mathrm{C}_{(2)}, \mathrm{C}_{(3)}, \mathrm{C}_{(4)}, \mathrm{C}_{(5)}, \mathrm{O}, \mathrm{N}_{(1)}, \mathrm{N}_{(2)}, \mathrm{N}_{(3)}, \mathrm{F}\right\}$; and $\Lambda_{11}=\left\{\mathrm{H}, \mathrm{C}_{(2)}, \mathrm{C}_{(3)}, \mathrm{C}_{(4)}, \mathrm{O}, \mathrm{N}_{(2)}, \mathrm{N}_{(3)}, \mathrm{S}_{(2)}, \mathrm{S}_{(4)}, \mathrm{S}_{(6)}, \mathrm{Cl}\right\}$, where $\mathrm{a}_{(i)}$ for a chemical element $a$ and an integer $i \geq 1$ means that a chemical element a with valence $i$.

- $\left|D_{\pi}\right|$ : the size of data set $D_{\pi}$ over $\Lambda$ for the property $\pi$.

- $\underline{n}, \bar{n}$ : the minimum and maximum values of the number $n(\mathbb{C})$ of nonhydrogen atoms in compounds $\mathbb{C}$ in $D_{\pi}$.

- $\underline{a}, \bar{a}$ : the minimum and maximum values of $a(\mathbb{C})$ for $\pi$ over compounds $\overline{\mathbb{C}}$ in $D_{\pi}$.

- $|\Gamma|$ : the number of different edge-configurations of interior-edges over the compounds in $D_{\pi}$.

- $|\mathcal{F}|$ : the number of non-isomorphic chemical rooted trees in the set of all 2-fringe-trees in the compounds in $D_{\pi}$. 
- $K$ : the number of descriptors in a feature vector $f(\mathbb{C})$.

Stage 2. We used the new feature function defined in our chemical model without suppressing hydrogen (see a full preprint version [22] for the detail). We standardize the range of each descriptor and the range $\{t \in \mathbb{R} \mid \underline{a} \leq t \leq \bar{a}\}$ of property values $a(\mathbb{C}), \mathbb{C} \in D_{\pi}$.

Stage 3. For each chemical property $\pi$, we select a penalty value $\lambda_{\pi}$ in the Lasso function from 36 different values from 0 to 100 by conducting linear regression as a preliminary experiment.

We conducted an experiment in Stage 3 to evaluate the performance of the prediction function based on cross-validation. For a property $\pi$, an execution of a cross-validation consists of five trials of constructing a prediction function as follows. First partition the data set $D_{\pi}$ into five subsets $D^{(k)}, k \in[1,5]$ randomly. For each $k \in[1,5]$, the $k$-th trial constructs a prediction function $\eta^{(k)}$ by conducting a linear regression with the penalty term $\lambda_{\pi}$ using the set $D_{\pi} \backslash D^{(k)}$ as a training data set. We used scikit-learn version 0.23.2 with Python 3.8.5 for executing linear regression with Lasso function. For each property, we executed ten crossvalidations and we show the median of test $\mathrm{R}^{2}\left(\eta^{(k)}, D^{(k)}\right), k \in[1,5]$ over all ten cross-validations. Recall that a subset of descriptors is selected in linear regression with Lasso function and let $K^{\prime}$ denote the average number of selected descriptors over all 50 trials. The running time per trial in a cross-validation was at most one second.

Table 1: Results in Phase 1.

\begin{tabular}{|c|c|c|c|c|c|c|c|c|c|}
\hline$\pi$ & $\Lambda$ & $D_{\pi} \mid$ & $\underline{n}, \bar{n}$ & $\underline{a}, \bar{a}$ & $|\Gamma||\mathcal{F}|$ & $K$ & $\lambda_{\pi}$ & $K^{\prime}$ & est $R^{2}$ \\
\hline Kow & $\Lambda_{2}$ & 68 & $\mathbf{T}$ & $-7.5,15.6$ & 251 & 2 & 5 & 80.3 & 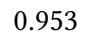 \\
\hline Kow & $\Lambda_{9}$ & 99 & 4,69 & & 37 & 35 & $5 E-5$ & 112.1 & 0.927 \\
\hline $\mathrm{Hc}$ & $\Lambda_{2}$ & 255 & 4,63 & 49.6, & 17106 & 154 & $1.9 \mathrm{E}-4$ & 19.2 & 0.946 \\
\hline $\mathrm{Hc}$ & $\Lambda_{7}$ & 282 & 4,63 & 9.6 & 21118 & 177 & $1.9 \mathrm{E}-4$ & 20.5 & 0.951 \\
\hline$V_{D}$ & $\Lambda_{2}$ & 474 & 4,30 & $0.7,20.6$ & 21160 & 214 & $1.0 \mathrm{E}-3$ & 3.6 & 0.927 \\
\hline VD & $\Lambda_{5}$ & 551 & 4,30 & 0.7 & 24191 & 0. & -4 & 8.0 & 0.942 \\
\hline OptR & $\Lambda_{2}$ & 147 & 5,44 & 0 & $21 \quad 55$ & 107 & $4.6 \mathrm{I}$ & 39.2 & 0.823 \\
\hline OptR & $\Lambda_{6}$ & 157 & 5,69 & $-117 .($ & $25 \quad 62$ & 1237 & 7.3 & 41.7 & 0.825 \\
\hline EDPA & $\Lambda_{1}$ & 2 & & & 933 & 64 & 1.0 & 10.9 & 999 \\
\hline MP & $\Lambda_{2}$ & 467 & 4,122 & $-185.33,3$ & 23142 & 1973 & $3.7 \mathrm{H}$ & 82.5 & 0.817 \\
\hline HA & $\Lambda_{3}$ & 115 & 4,11 & $1100.6,3009.6$ & $8 \quad 83$ & 1153 & $3.7 \mathrm{H}$ & 39.0 & 0.997 \\
\hline $\mathrm{HF}$ & $\Lambda_{1}$ & 82 & 4,16 & $30.2,94.8$ & $5 \quad 50$ & 74 & $1.0 \mathrm{H}$ & 34.0 & 0.987 \\
\hline U0 & $\Lambda_{10}$ & 977 & 4,9 & $-570.6,-272.8$ & 59190 & 297 & 1.0 & 246.7 & 0.999 \\
\hline Lumo & $\Lambda_{10}$ & 977 & & $-0.11,0$ & 59190 & 297 & $6.4 \mathrm{~F}$ & 133.9 & .841 \\
\hline ALPHA & $\Lambda_{10}$ & 977 & 4,9 & 6 & 59190 & 297 & 1.0 & 125.5 & 0.961 \\
\hline $\mathrm{Cv}$ & $\Lambda_{10}$ & 977 & & 19.2 & 59190 & 297 & $1.0 \mathrm{I}$ & 165.3 & 0.961 \\
\hline $\mathrm{SL}_{\mathrm{L}}$ & $\Lambda_{9}$ & 915 & 4,55 & -11. & 42207 & 3007 & $7.3 \mathrm{E}-5$ & 130.6 & 0.808 \\
\hline SFT & $\Lambda_{4}$ & 247 & 5 & 12.3 & 1191 & 128 & $6.4 \mathrm{H}$ & 20.9 & 0.804 \\
\hline VIS & $\Lambda_{4}$ & 282 & 5,36 & $-0.64,1.63$ & 1288 & 126 & $8.2 \mathrm{E}-4$ & 16.3 & 0.893 \\
\hline IHCLIQ & $\Lambda_{2}$ & 770 & 4,78 & 956.1 & 23200 & 256 & $1.9 \mathrm{E}-5$ & 82.2 & 0.987 \\
\hline ICLIQ & $\Lambda_{7}$ & 865 & 4,7 & $106.3,1956.1$ & 29246 & 316 & $8.2 \mathrm{E}-6$ & 139.1 & 0.986 \\
\hline IHCSOL & $\Lambda_{8}$ & 581 & 5,70 & $67.4,1220.9$ & 33124 & 192 & $2.8 \mathrm{E}-5$ & 75.9 & 0.985 \\
\hline HCSOL & $\Lambda_{11}$ & 668 & 5,70 & 220.9 & 40140 & 228 & $2.8 \mathrm{E}-5$ & 86.7 & 0.982 \\
\hline LP & $\Lambda_{2}$ & 615 & 6,60 & $-3.62,6.84$ & 32116 & 186 & $1.0 \mathrm{E}-4$ & 98.5 & 0.856 \\
\hline LP & $\Lambda_{9}$ & 936 & 6,74 & $-3.62,6.84$ & 44136 & 231 & $6.4 \mathrm{E}-5$ & 130.4 & 0.840 \\
\hline
\end{tabular}

Table 1 shows the results on Stages 2 and 3, where we denote the following:

- $\lambda_{\pi}$ : the penalty value in the Lasso function selected for a property $\pi$, where $a \mathrm{E} b$ means $a \times 10^{b}$.

- $K^{\prime}$ : the average of the number of descriptors selected in the linear regression over all 50 trials in ten cross-validations.

- test $\mathrm{R}^{2}$ : the median of test $\mathrm{R}^{2}$ over all 50 trials in ten cross-validations.

Recall that the adjacency-configuration for leaf-edges was introduced as a new descriptor in this paper. Without including this new descriptor, the test $\mathrm{R}^{2}$ for property VIS was 0.790 , that for Lumo was 0.799 and that for MP was 0.796 , while the test $\mathrm{R}^{2}$ for each of the other properties in Table 1 was almost the same.

From Table 1, we observe that a relatively large number of properties admit a good prediction function based on linear regression. The number $K^{\prime}$ of descriptors used in linear regression is considerably small for some properties.

Results on Phase 2. To execute Stages 4 and 5 in Phase 2, we used a set of seven instances $I_{\mathrm{a}}, I_{\mathrm{b}}^{i}, i \in[1,4], I_{\mathrm{c}}$ and $I_{\mathrm{d}}$ based on seed graphs prepared by Shi et al. [17]. We here present their seed graphs $G_{C}$ (see a full preprint version [22] for the details of instances $I_{\mathrm{a}}, I_{\mathrm{b}}^{i}, i \in[1,4], I_{\mathrm{c}}$ and $I_{\mathrm{d}}$ ). The seed graph $G_{\mathrm{C}}$ of instance $I_{\mathrm{a}}$ is given by the graph in Figure 4(a). The seed graph $G_{\mathrm{C}}^{1}$ (resp., $\left.G_{\mathrm{C}}^{i}, i=2,3,4\right)$ of instances $I_{\mathrm{b}}^{1}$ and $I_{\mathrm{d}}\left(\right.$ resp., $\left.I_{\mathrm{b}}^{i}, i=2,3,4\right)$ is illustrated in Figure 5.

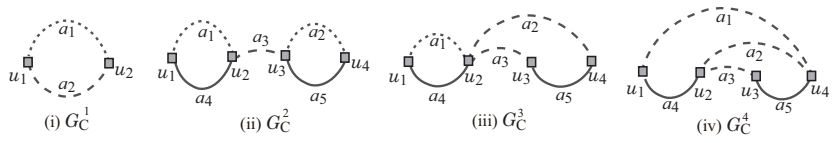

Figure 5: (i) Seed graph $G_{\mathrm{C}}^{1}$ for $I_{\mathrm{b}}^{1}$ and $I_{\mathrm{d}}$; (ii) Seed graph $G_{\mathrm{C}}^{2}$ for $I_{\mathrm{b}}^{2}$; (iii) Seed graph $G_{\mathrm{C}}^{3}$ for $I_{\mathrm{b}}^{3}$; (iv) Seed graph $G_{\mathrm{C}}^{4}$ for $I_{\mathrm{b}}^{4}$.

Instance $I_{\mathrm{c}}$ has been introduced in order to infer a chemical graph $\mathbb{C}^{\dagger}$ such that the core of $\mathbb{C}^{\dagger}$ is equal to the core of chemical graph $\mathbb{C}_{A}$ : CID 24822711 in Figure 6(a) and the frequency of each edge-configuration in the non-core of $\mathbb{C}^{\dagger}$ is equal to that of chemical graph $\mathbb{C}_{B}$ : CID 59170444 in Figure 6(b). This means that the seed graph $G_{C}$ of $I_{\mathrm{c}}$ is the core of $\mathbb{C}_{A}$ which is indicated by a shaded area in Figure 6(a).

Instance $I_{\mathrm{d}}$ has been introduced in order to infer a chemical monocyclic graph $\mathbb{C}^{\dagger}$ such that the frequency vector of edge-configurations in $\mathbb{C}^{\dagger}$ is a vector obtained by merging those of chemical graphs $\mathbb{C}_{A}$ : CID 10076784 and $\mathbb{C}_{B}$ : CID 44340250 in Figure 6(c) and (d), respectively.

Stage 4. We executed Stage 4 for five properties $\pi \in\left\{H_{C}, V_{D}, O_{p t}\right.$, IHCLIQ, Vis\}.

For the MILP formulation $\mathcal{M}\left(x, y ; C_{1}\right)$ in Section 4 , we use the prediction function $\eta_{w, b}$ that attained the median test $\mathrm{R}^{2}$ in Table 1 . To solve an MILP in Stage 4, we used CPLEX version 12.10. Table 2 shows the computational results of the experiment in Stage 4 for property Hc, where we denote the following:

- $y^{*}, \bar{y}^{*}:$ lower and upper bounds $y^{*}, \bar{y}^{*} \in \mathbb{R}$ on the value $a(\mathbb{C})$ of a chemical graph $\mathbb{C}$ to be inferred;

- \#v (resp., \#c): the number of variables (resp., constraints) in the MILP in Stage 4; 


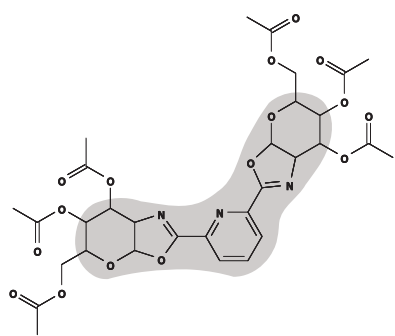

(a) $\mathbb{C} A$ : CID 24822711

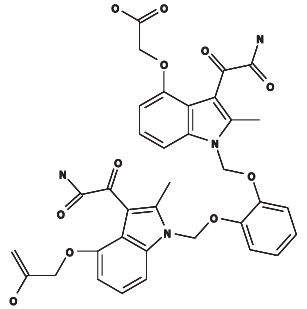

(b) $\mathbb{C}_{B}$ : CID 59170444

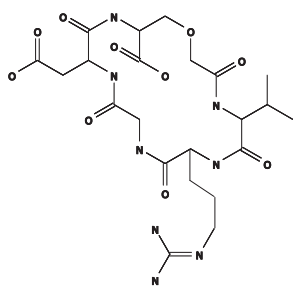

(c) $\mathbb{C} A$ : CID 10076784

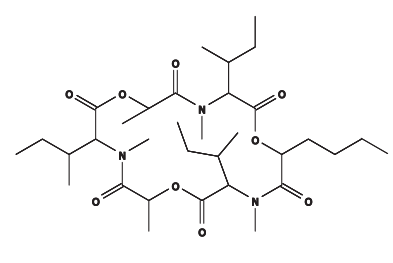

(d) $\mathbb{C} B$ : CID 44340250
Figure 6: An illustration of chemical compounds for instances $I_{\mathrm{c}}$ and $I_{\mathrm{d}}:$ (a) $\mathbb{C}_{A}:$ CID 24822711; (b) $\mathbb{C}_{B}$ : CID 59170444; (c) $\mathbb{C}_{A}$ : CID 10076784; (d) $\mathbb{C}_{B}$ : CID 44340250, where hydrogens are omitted.

- I-time: the time (sec.) to solve the MILP in Stage 4;

- $n$ : the number $n\left(\mathbb{C}^{\dagger}\right)$ of non-hydrogen atoms in the chemical graph $\mathbb{C}^{\dagger}$ inferred in Stage 4; and

- $\mathrm{n}^{\text {int }}$ : the number $\mathrm{n}^{\text {int }}\left(\mathbb{C}^{\dagger}\right)$ of interior-vertices in the chemical graph $\mathbb{C}^{\dagger}$ inferred in Stage 4;

- $\eta\left(f\left(\mathbb{C}^{\dagger}\right)\right)$ : the predicted property value $\eta\left(f\left(\mathbb{C}^{\dagger}\right)\right)$ of the chemical graph $\mathbb{C}^{\dagger}$ inferred in Stage 4.

Table 2: Results of Stages 4 and 5 for Hc.

\begin{tabular}{ccrrrrrrrrrrr}
\hline inst. & $\underline{y}^{*}, \bar{y}^{*}$ & \#v & \multicolumn{3}{c}{ \#c } & I-time & $n \mathrm{n}^{\text {int }}$ & $\eta\left(f\left(\mathbb{C}^{\dagger}\right)\right)$ & D-time & $\mathbb{C}$-LB & $\# \mathbb{C}$ \\
\hline$I_{\mathrm{a}}$ & 5950,6050 & 9902 & 9255 & 4.6 & 44 & 25 & 5977.9 & 0.068 & 1 & 1 \\
$I_{\mathrm{b}}^{1}$ & 5950,6050 & 9404 & 6776 & 1.7 & 36 & 10 & 6007.1 & 0.048 & 6 & 6 \\
$I_{\mathrm{b}}^{2}$ & 5950,6050 & 11729 & 9891 & 16.7 & 50 & 25 & 6043.7 & 38.7 & $2.4 \mathrm{E} 5$ & 100 \\
$I_{\mathrm{b}}^{3}$ & 5950,6050 & 11510 & 9894 & 16.3 & 47 & 25 & 6015.4 & 0.353 & 8724 & 100 \\
$I_{\mathrm{b}}^{4}$ & 5950,6050 & 11291 & 9897 & 9.0 & 49 & 26 & 5971.6 & 0.304 & 84 & 84 \\
$I_{\mathrm{c}}$ & 13700,13800 & 6915 & 7278 & 0.7 & 50 & 33 & 13703.3 & 0.016 & 1 & 1 \\
$I_{\mathrm{d}}$ & 13700,13800 & 5535 & 6781 & 4.9 & 44 & 23 & 13704.7 & 0.564 & $4.3 \mathrm{E} 5$ & 100 \\
\hline
\end{tabular}

From the computational results (see a full preprint version [22] for the results of the other properties $\pi \in\{\mathrm{VD}$, OptR, IHCLIQ, VIs $\}$ ), we observe that an instance with a large number of variables and constraints takes more running time than those with a smaller size in general. In this experiment, we prepared several different types of instances: instances $I_{\mathrm{a}}$ and $I_{\mathrm{c}}$ have restricted seed graphs, the other instances have abstract seed graphs and instances $I_{\mathrm{c}}$ and $I_{\mathrm{d}}$ have restricted set of fringe-trees. All instances in this experiment are solved in a few seconds to around 30 seconds with our MILP formulation.

Stage 5. We executed Stage 5 to generate a more number of target chemical graphs $\mathbb{C}^{*}$, where we call a chemical graph $\mathbb{C}^{*}$ a chemical isomer of a target chemical graph $\mathbb{C}^{\dagger}$ of a topological specification $\sigma$ if $f\left(\mathbb{C}^{*}\right)=f\left(\mathbb{C}^{\dagger}\right)$ and $\mathbb{C}^{*}$ also satisfies the same topological specification $\sigma$. We computed chemical isomers $\mathbb{C}^{*}$ of each target chemical graph $\mathbb{C}^{\dagger}$ inferred in Stage 4. We execute an algorithm for generating chemical isomers of $\mathbb{C}^{\dagger}$ up to 100 when the number of all chemical isomers exceeds 100. Such an algorithm can be obtained from the dynamic programming proposed by Tanaka et al. [18] with a slight modification. The algorithm first decomposes $\mathbb{C}^{\dagger}$ into a set of acyclic chemical graphs, next replaces each acyclic chemical graph $T$ with another acyclic chemical graph $T^{\prime}$ that admits the same feature vector as that of $T$, and finally assembles the resulting acyclic chemical graphs into a chemical isomer $\mathbb{C}^{*}$ of $\mathbb{C}^{\dagger}$. The algorithm can compute a lower bound on the total number of all chemical isomers of $\mathbb{C}^{\dagger}$ without generating all of them.

Table 2 shows the computational results of the experiment in Stage 5 for property Hc, where we denote the following:

- D-time: the running time (sec.) to execute the dynamic programming algorithm in Stage 5 to compute a lower bound on the number of all chemical isomers $\mathbb{C}^{*}$ of $\mathbb{C}^{\dagger}$ and generate all (or up to 100) chemical isomers $\mathbb{C}^{*}$;

- $\mathbb{C}$-LB: a lower bound on the number of all chemical isomers $\mathbb{C}^{*}$ of $\mathbb{C}^{\dagger}$; and

- \#C: the number of all (or up to 100) chemical isomers $\mathbb{C}^{*}$ of $\mathbb{C}^{\dagger}$ generated in Stage 5.

From Table 2, we observe that the running time for generating up to 100 target chemical graphs in Stage 5 is less than 0.4 second for many cases.

\section{CONCLUDING REMARKS}

In the previous applications of the framework of inferring chemical graphs, artificial neural network (ANN) and decision tree have been used for the machine learning of Stage 3. In this paper, we used linear regression in Stage 3 for the first time and derived an MILP formulation that simulates the computation process of linear regression. We also extended a way of specifying a target value $y^{*}$ in a property so that the predicted value $\eta\left(f\left(\mathbb{C}^{\dagger}\right)\right)$ of a target chemical graph $\mathbb{C}^{\dagger}$ is required to belong to an interval between two specified values $\underline{y}^{*}$ and $\bar{y}^{*}$. In this paper, we modified a model of chemical compounds so that multi-valence chemical elements, cation and anion are treated, and introduced the rank and the adjacencyconfiguration of leaf-edges as new descriptors in a feature vector of a chemical graph. We implemented the new system of the framework and conducted computational experiments for Stages 1 to 5 . We found 18 properties for which linear regression delivers a relatively good prediction function by using our feature vector based on the two-layered model. We also observed that an MILP formulation for inferring a chemical graph in Stage 4 can be solved efficiently over different types of test instances with complicated topological specifications. The experimental result suggests that our method can infer chemical graphs with up to 50 non-hydrogen atoms. 


\section{REFERENCES}

[1] T. Akutsu and H. Nagamochi. 2019. A mixed integer linear programming formulation to artificial neural networks. In Proc. of the 2nd Int. Conf. on Information Science and Systems. $215-220$.

[2] N. A. Azam, R. Chiewvanichakorn, F. Zhang, A. Shurbevski, H. Nagamochi, and T. Akutsu 2020. A method for the inverse QSAR/QSPR based on artificial neural networks and mixed integer linear programming. In Proc. of the 13th International foint Conference on Biomedical Engineering Systems and Technologies - Volume 3: BIOINFORMATICS. 101-108.

[3] R. S. Bohacek, C. McMartin, and W. C. Guida. 1996. The art and practice of structure-based drug design: A molecular modeling perspective. Med. Res. Rev. 16 (1996), 3-50.

[4] N. De Cao and T. Kipf. 2018. MolGAN: An implicit generative model for small molecular graphs. (2018). arXiv:1805.11973

[5] F. Ghasemi, A. Mehridehnavi, A. Pérez-Garrido, and H. Pérez-Sánchez. 2018. Neural network and deep-learning algorithms used in QSAR studies: merits and drawbacks. Drug Discovery Today 23 (2018), 1784-1790.

[6] R. Gómez-Bombarelli, J. N. Wei, D. Duvenaud, J. M. Hernández-Lobato, B. SánchezLengeling, D. Sheberla, J. Aguilera-Iparraguirre, T. D. Hirzel, R. P. Adams, and A. AspuruGuzik. 2018. Automatic chemical design using a data-driven continuous representation of molecules. ACS Cent. Sci. 4 (2018), 268-276.

[7] A Hoerl and R Kennard. 1988. Ridge regression, in 'encyclopedia of statistical sciences', vol. 8.

[8] H. Ikebata, K. Hongo, T. Isomura, R. Maezono, and R. Yoshida. 2017. Bayesian molecular design with a chemical language model. 7. Comput. Aided Mol. Des. 31 (2017), 379-391.

[9] T. N. Kipf and M. Welling. 2016. Semi-supervised classification with graph convolutional networks. (2016). arXiv:1609.02907

[10] M. J. Kusner, B. Paige, and J. M. Hernández-Lobato. 2017. Grammar variational autoencoder. In Proc. of the 34th International Conference on Machine Learning-Volume 70 1945-1954.
[11] Y-C. Lo, S. E. Rensi, W. Torng, and R. B. Altman. 2018. Machine learning in chemoinformatics and drug discovery. Drug Discovery Today 23 (2018), 1538-1546.

[12] K. Madhawa, K. Ishiguro, K. Nakago, and M. Abe. 2019. GraphNVP: an invertible flow model for generating molecular graphs. (2019). arXiv:1905.11600

[13] T. Miyao, H. Kaneko, and K. Funatsu. 2016. Inverse QSPR/QSAR analysis for chemical structure generation (from y to x). F. Chem. Inf. Model. 56 (2016), 286-299.

[14] C. Rupakheti, A. Virshup, W. Yang, and D. N. Beratan. 2015. Strategy to discover diverse optimal molecules in the small molecule universe. F. Cheminformatics 55 (2015), 529-537.

[15] M. H. S. Segler, T. Kogej, C. Tyrchan, and M. P. Waller. 2017. Generating focused molecule libraries for drug discovery with recurrent neural networks. ACS Cent. Sci. 4 (2017), $120-131$.

[16] C. Shi, M. Xu, Z. Zhu, W. Zhang, M. Zhang, and J. Tang. 2020. GraphAF: a flow-based autoregressive model for molecular graph generation. (2020). arXiv:2001.09382

[17] Y. Shi, J. Zhu, N. A. Azam, K. Haraguchi, L. Zhao, H. Nagamochi, and T. Akutsu. 2021 An inverse QSAR method based on a two-layered model and integer programming International fournal of Molecular Sciences 22 (2021), 2847.

[18] K. Tanaka, J. Zhu, N. A. Azam, K. Haraguchi, L. Zhao, H. Nagamochi, and T. Akutsu. 2021. An inverse QSAR method based on decision tree and integer programming. In The 17th International Conference on Intelligent Computing. August in Shenzhen, China (to appear) $12-15$.

[19] I. V. Tetko and O. Engkvist. 2020. From Big Data to Artificial Intelligence: chemoinformatics meets new challenges. F. Cheminformatics 12 (2020), 74.

[20] Robert Tibshirani. 1996. Regression shrinkage and selection via the lasso. Fournal of the Royal Statistical Society: Series B (Methodological) 58, 1 (1996), 267-288.

[21] X. Yang, J. Zhang, K. Yoshizoe, K. Terayama, and K. Tsuda. 2017. ChemTS: an efficient python library for de novo molecular generation. STAM 18 (2017), 972-976.

[22] J. Zhu, N. A. Azam, K. Haraguchi, L. Zhao, H. Nagamochi, and T. Akutsu. 2021. A method for molecular design based on linear regression and integer programming. (2021) arXiv:2107.02381 